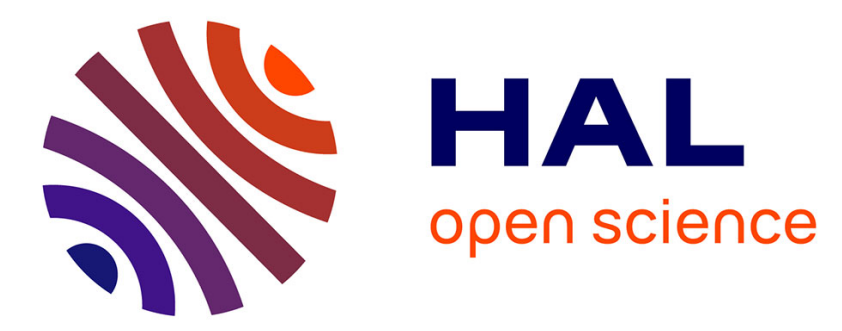

\title{
Les restructurations à l'usine Perrier, ou quand deux " cultures d'entreprise " s'affrontent sur le lieu de travail (1990-2000)
}

Irène Favier

\section{- To cite this version:}

Irène Favier. Les restructurations à l'usine Perrier, ou quand deux " cultures d'entreprise " s'affrontent sur le lieu de travail (1990-2000). Le Mouvement social, 2009, 228 (3), pp.147 - 162. 10.3917/lms.228.0147 . hal-01744394

\section{HAL Id: hal-01744394 \\ https://hal.science/hal-01744394}

Submitted on 27 Mar 2018

HAL is a multi-disciplinary open access archive for the deposit and dissemination of scientific research documents, whether they are published or not. The documents may come from teaching and research institutions in France or abroad, or from public or private research centers.
L'archive ouverte pluridisciplinaire HAL, est destinée au dépôt et à la diffusion de documents scientifiques de niveau recherche, publiés ou non, émanant des établissements d'enseignement et de recherche français ou étrangers, des laboratoires publics ou privés. 


\title{
LES RESTRUCTURATIONS À L'USINE PERRIER, OU QUAND DEUX «CULTURES D'ENTREPRISE » S'AFFRONTENT SUR LE LIEU DE TRAVAIL (1990-2000) \\ Irène Favier
}

\author{
La Découverte | «Le Mouvement Social »
}

2009/3 n²28 | pages 147 à 162

ISSN 0027-2671

ISBN 9782707158116

Article disponible en ligne à l'adresse :

https://www.cairn.info/revue-le-mouvement-social-2009-3-page-147.htm

\section{Pour citer cet article :}

Irène Favier, « Les restructurations à l'usine Perrier, ou quand deux « cultures d'entreprise »s'affrontent sur le lieu de travail (1990-2000) », Le Mouvement Social 2009/3 (n²28), p. 147-162.

DOI 10.3917/lms.228.0147

Distribution électronique Cairn.info pour La Découverte.

(C) La Découverte. Tous droits réservés pour tous pays.

La reproduction ou représentation de cet article, notamment par photocopie, n'est autorisée que dans les limites des conditions générales d'utilisation du site ou, le cas échéant, des conditions générales de la licence souscrite par votre établissement. Toute autre reproduction ou représentation, en tout ou partie, sous quelque forme et de quelque manière que ce soit, est interdite sauf accord préalable et écrit de l'éditeur, en dehors des cas prévus par la législation en vigueur en France. Il est précisé que son stockage dans une base de données est également interdit. 


\title{
Les restructurations à l'usine Perrier, ou quand deux « cultures d'entreprise " s'affrontent sur le lieu de travail (1990-2000)
}

par Irène FAVIER ${ }^{*}$

\begin{abstract}
A u cours de la décennie 1990, une série de restructurations furent menées à l'usine de la Source Perrier, suite à son rachat par le groupe Nestlé. Les multiples changements générés sur le site gardois furent considérables : en dix ans, $40 \%$ des 2500 emplois furent supprimés. Jusqu'alors relativement localisée, la sphère au sein de laquelle les acteurs sociaux évoluaient s'en trouva élargie à l'échelle internationale : les interlocuteurs des salariés n'étaient plus les représentants de l'ancienne maison-mère située à Vergèze (Gard), mais une équipe de direction coutumière d'une gestion mondialisée des affaires, et dont le siège social se trouvait désormais en Suisse. Décidées à distance, à l'aune de critères globaux, et dans un contexte de difficultés économiques, les vagues de restructuration du groupe Perrier ont donné lieu à un durcissement et une modification notables des rapports sociaux sur le site. Les luttes sociales y firent l'objet de mises en discours bien particulières et de pratiques militantes renouvelées : tandis que la direction promouvait un "nouvel esprit » auprès des salariés, le syndicat majoritaire CGT-Perrier se réclamait, à des fins de lutte sociale, de la véritable « culture d'entreprise " propre à Perrier.

Faite de soubresauts et d'accalmies, la décennie 1990 apparaît donc comme une période cruciale, au cours de laquelle s'est opérée une mutation majeure dans les techniques de gestion de la main-d'œuvre - basculement dont l'ampleur nous semble comparable aux mutations opérées par le patronat français dans les années 1880 et $1920^{1}$. Un management d'inspiration paternaliste a en effet laissé place à des méthodes managériales mondialisées : à la faveur de ces restructurations se sont jouées localement la substitution d'un type de capitalisme à un autre et l'introduction de nouvelles méthodes de travail. La réduction drastique des effectifs à la Source Perrier s'insère dans un phénomène plus large de désindustrialisation aux conséquences majeures sur le tissu social et politique ; dans certains secteurs le capitalisme contemporain semble désormais arrivé à un stade où les effectifs salariés déclinent.
\end{abstract}

Le présent article souhaite montrer que, loin de se réduire à une question technique, les restructurations chez Perrier constituent un fait social multiforme, ayant affecté tant les salariés désormais exclus de l'usine que ceux qui continuent à y travailler. Il se fonde sur les comptes rendus du Comité d'établissement et du

* Historienne, élève de l'École Normale Supérieure.

1. G. Noiriel, " Du "patronage" au "paternalisme" : la restructuration des formes de domination de la main-d'œuvre ouvrière dans l'industrie métallurgique française ", Le Mouvement Social, juilletseptembre 1988, p. 17-35. Signalons depuis notamment J. Fombonne, Personnel et DRH : l'affirmation de la fonction personnel dans les entreprises (France, 1830-1990), Paris, Vuibert, 2001 et P.-D. GaLloro, Ouvriers du fer, princes du vent : histoire des flux de main-d'ouvre dans la sidérurgie lorraine, 1880-1939, Metz, Éditions Serpenoise, 2001. 
Comité d'hygiène, de sécurité et des conditions de travail, sur des entretiens avec des ouvriers, des délégués du personnel et des syndicalistes CGT et CGC, et sur la presse $^{2}$. Après avoir exposé le déroulement des restructurations proprement dites, on se propose ainsi de mettre en lumière deux phénomènes majeurs qui suggèrent la profondeur des bouleversements survenus durant cette décennie heurtée : la place croissante du droit dans les relations et les conflits sociaux, puis la redéfinition des identités et la concurrence des mémoires sur le site.

\section{Une décennie de restructurations}

La décennie 1990 s'ouvre, pour le groupe Perrier et ses salariés, sur un retournement brutal de conjoncture économique et sur une âpre bataille économique et juridique. Ces bouleversements sont occasionnés par ce que les acteurs en présence nomment presque immédiatement "l'affaire du benzène ». Un laboratoire américain d'Atlanta ayant détecté des traces de cet agent cancérogène dans une bouteille de Perrier soumise à l'analyse, le PDG du groupe, Gustave Leven, décide de procéder au retrait rapide de la totalité des 160 millions de bouteilles alors commercialisées sur le marché américain. Cette crise occasionne une perte considérable de parts de marché, une baisse brutale du volume de production, ainsi qu'une chute du cours de l'action Perrier. Elle facilite l'OPA lancée par le groupe Nestlé qui, au terme d'une lutte juridique, finit par acquérir le groupe Perrier, après avoir obtenu l'aval de la Commission européenne en 1992. Dès l'arrivée de la nouvelle équipe dirigeante sur le site, la restructuration du site est à l'ordre du jour : leitmotiv des discours adressés à l'attention des actionnaires, des acteurs sociaux et de la presse, elle est présentée comme un impératif préalable à toute véritable relance commerciale.

Le terme même de restructuration fait débat et appelle une précision terminologique. Il s'agit de la réorganisation structurelle d'une entité économique et juridique réalisée en vue d'aboutir à une baisse des coûts de production. Mais l'usage du mot a varié depuis les années 1970. Jusqu'alors les restructurations industrielles étaient survenues dans des secteurs dont la pérennité avait été sérieusement remise en cause par le progrès technique, tels que les charbonnages, la sidérurgie, le textile, ou les chantiers navals. Depuis lors, la restructuration des sites ou entreprises " en difficulté " - pour reprendre une terminologie juridique complexe et discutée - fait figure d'« outil permanent d'adaptation industrielle des entreprises, à la recherche d'une compétitivité croissante, qui, en outre, est souvent pensée à une échelle transnationale, tandis que jusqu'aux années 1970, les restructurations industrielles désignaient des phénomènes bien identifiés : elles concernaient un petit nombre de secteurs industriels dont l'adaptation paraissait douloureuse, mais inéluctable ${ }^{3}$. Dans le cas de Perrier, les restructurations interviennent au contraire dans un contexte d'expansion du marché des eaux minérales embouteillées, marqué par des taux de profit importants, et qui justifient l'attention que le rachat de Perrier a suscitée chez des " géants " économiques tels que Nestlé et Danone ${ }^{4}$. Ces restructurations appellent dès lors une interprétation radicalement différente de celles opérées dans les secteurs

2. Pour le détail des sources utilisées, voir l'ouvrage cité à la note 29 .

3. F. Aggeri et F. Pallez, « Restructurations, délocalisations : les nouvelles formes de l'action territoriale ", Revue de l'IRES, n 47, 2005, p. 235-256.

4. D. Spar et K. Bebenek, "Profitable springs: the rise, sources, and structures of the bottled water business", Entreprises et Histoire, avril 2008, p. 100-118. 
en crise structurelle durant les années 1970 : elles apparaissent comme une réponse à la financiarisation du capitalisme contemporain et au haut degré de compétition qui caractérise ce secteur ${ }^{5}$.

D'autre part, les opérations que recouvre la notion de restructuration sont diverses. Leur manifestation juridique en est certes la plus visible. Dans le cas Perrier, les restructurations ont visé le site de Vergèze à deux titres : non seulement en tant qu'unité de production, mais aussi en tant que siège social de la maison-mère de l'ancienne Société Générale des Grandes Sources. Toutefois les restructurations chez Perrier ne se limitent ni à une reconfiguration juridique à l'échelle du groupe ni à une redistribution des hiérarchies. La suppression drastique des effectifs en est sans doute la caractéristique majeure ; mais il importe de prendre également en compte les bouleversements affectant le personnel restant sur le site après ces départs massifs. Le terme de restructuration recouvre donc un ensemble de mesures réalisées au sein de l'espace et de la communauté de travail, dont il s'agit à présent de souligner trois modalités principales.

\section{La réduction des effectifs et sa justification : le renouvellement du discours patronal}

Les restructurations prirent avant tout la forme de trois " plans sociaux " successifs, destinés à réduire les effectifs, et réalisés selon des intervalles de temps relativement réguliers $(1993,1995,1998)$. Au terme de la décennie, un millier des 2500 emplois existant en 1989 ont été supprimés selon des moyens divers, en évitant toutefois de préférence, pour des raisons de communication et d'image notamment, les procédures juridiques de licenciements économiques. Ainsi les plans visant la baisse des effectifs consistèrent-ils tout d'abord en des mesures de départs en retraite anticipés - démarche rendue possible par l'intervention de la puissance publique au titre du Fonds National pour l'Emploi (FNE). Les responsables des ressources humaines aménagèrent également des dispositifs de réduction individuelle du temps de travail, certains salariés optant pour du travail à temps partiel volontaire ou pour du travail intermittent. Ils proposèrent également des primes de départ, surnommées " primes à la valise ", et qui étaient accompagnées de mesures dites de plans de redynamisation du bassin d'emploi de Nîmes. La réduction du nombre d'emplois sur le site de Vergèze fut de plus réalisée par le biais de mesures d'externalisation des fonctions de gardiennage ou de nettoyage. Enfin, les dirigeants furent suspectés d'envisager une opération de délocalisation de la production, après que les acteurs sociaux locaux eurent pris connaissance de la production en Egypte par Nestlé de bouteilles contenant de l'eau gazeuse et dont la forme ressemblait à plus d'un titre à celle de la bouteille Perrier.

Cette baisse conséquente du nombre d'emplois, sur un site dont l'essor considérable depuis l'après-guerre s'était traduit par des embauches régulières et massives, imposait aux dirigeants d'appuyer leurs pratiques et leurs décisions sur un dispositif idéologique renouvelé, fournissant des justifications à leurs " partenaires sociaux ». Par exemple, les dirigeants du site firent à maintes reprises référence à un critère numérique, le ratio " nombre de cols ${ }^{6}$ produits/personnes/an ». L'objectif était fixé

5. R. Brenner, Economics of global turbulence, Londres, Verso, 2006 ; The Boom and the Bubble, Londres, Verso, 2002.

6. Terme technique synonyme de bouteille. 
à 800000 : estimé au moyen d'une comparaison avec le site de production des bouteilles San Pellegrino (acquis en 1997), il était bien supérieur à la moyenne en vigueur sur le site de Vergèze, et ce pour des raisons structurelles ${ }^{7}$. Un tel outil faisait ainsi figure, aux yeux de ses concepteurs, de critère " objectif ", car il était chiffré, justifiant les restructurations. Bien qu'elles se soient par la suite révélées incapables de garantir la paix sociale sur le site en évitant les conflits sociaux et d'assurer aux yeux du public une image positive pour le groupe et ses marques, ces justifications traduisent le renouvellement dont les discours patronaux ont fait l'objet depuis les années $1990^{8}$. Dans son ouvrage Principes simples pour diriger dans un monde complexe $^{9}$, Helmut Maucher, alors PDG du groupe Nestlé, se propose ainsi d'éclairer son lecteur sur la "philosophie » inspirant les décisions prises par les dirigeants du groupe, et de fournir aux cadres de Nestlé quelques repères afin de mener à bien les missions qui leur sont confiées.

Il s'avère utile d'appréhender ce discours dans une perspective historique. Les mesures d'externalisation des activités non directement liées à la production sont, par exemple, justifiées au nom d'un impératif dit de " recentrage sur le cœur de métier ». Si pour le juriste ce type de discours relance l'épineux problème de la définition juridique du terme d'entreprise ${ }^{10}$, il illustre pour l'historien un tournant majeur opéré en termes d'élaboration des stratégies industrielles. En effet, le phénomène de concentration industrielle auquel ont donné lieu les stratégies d'intégration verticale au cours du Xx ${ }^{\mathrm{e}}$ siècle ${ }^{11}$ semble laisser place à un démembrement, au moins juridique, des entités usinières. L'impact juridique et social de ces mesures est considérable ; elles apparaissent comme un phénomène majeur depuis la crise des années 1970. Ainsi, au nom de l'impératif de baisse des coûts de production, les grandes entreprises reportent un certain nombre de leurs coûts fixes sur un tissu de sous-traitants environnants, qui assurent des rémunérations sensiblement inférieures à la moyenne en vigueur dans les grandes entreprises. Cette stratégie d'externalisation permet dès lors d'opérer une sélection progressive des emplois les moins productifs.

\section{Réaménagements à l'échelle du groupe Nestlé Waters et reconfiguration du site de production : nouvelles structures, nouveaux espaces}

L'organigramme du groupe Perrier fut entièrement refondu à la suite du rachat : l'établissement de Vergèze était désormais inclus dans le pôle eaux minérales du groupe Nestlé, Nestlé Waters. L’ancienne Société Générale des Grandes Eaux (SGGE) cessait d'être, certaines sources étant revendues, d'autres incluses à leur tour dans la nouvelle structure juridique. L'ancienne Société Générale des Grandes Sources regroupait plusieurs autres unités de production, telles que Vichy, Thonon ou Vittel. $S^{\prime} y$ adjoignait également le groupe Perrier of America - véritable motivation du

7. Le site de production de San Pellegrino n'était en effet pas caractérisé par l'internalisation d'activités connexes telles que le gardiennage, la surveillance, ou l'entretien. Une plus grande proportion d'employés étant affectés à la production, le ratio de productivité par tête y était par conséquent bien plus élevé.

8. E. Chiapello et L. Boltanski, Le nouvel esprit du capitalisme, Paris, Gallimard, 1999.

9. Paris, Maxima, 1995.

10. G. Lyon-CaEn, "Que sait-on de plus sur l'entreprise?", in Mélanges en l'honneur du Président M. Despax, Toulouse, Presses de l'Université des sciences sociales, 2002, p. 33. J.-P. Robé, "À qui appartiennent les entreprises ? ", Le Débat, mai-août 2009, p. 32-36.

11. P. Fridenson, Histoire des usines Renault, t. I, $2^{\mathrm{e}}$ éd., Paris, Le Seuil, 1998. 
rachat par Nestlé, qui désirait pénétrer le marché américain. La SGGS étant juridiquement absorbée par le groupe Nestlé, l'ancienne maison-mère située à Vergèze se voyait désormais attribuer le simple rôle de site de production. Loin de constituer un simple changement "sur le papier", cette refonte hiérarchique eut une profonde signification pour les salariés, notamment pour les cadres : de la maison-mère placée au centre de gravité d'un groupe, la Source Perrier devenait un simple établissement devant rendre compte de ses résultats à des supérieurs hiérarchiques localisés ailleurs qu'à Vergèze. Pour autant, on ne saurait interpréter les relations entre le nouveau centre décisionnel et l'établissement vergèzois de façon unilatérale. La nouvelle hiérarchie en vigueur est certes dépourvue d'ambiguïté et la distribution du pouvoir au sein des instances décisionnelles est claire : les consignes viennent de Paris ou de Vevey, et sont appliquées à Vergèze. Pour autant, dans le domaine de l'ingénierie des eaux minérales, les relations qui s'établissent entre le nouveau centre suisse et l'ancien centre vergézois sont complexes : les échanges réalisés correspondent, cette fois, moins à un modèle pyramidal qu'à une logique de "transfert culturel " ${ }^{12}$. Les compétences dans ce domaine se trouvaient en effet à Vergèze, non chez Nestlé qui, bien que mû par la volonté de pénétrer le marché florissant des eaux minérales, ne pouvait pas s'appuyer sur une expérience précédente dans ce secteur. Nombreux furent ainsi les ingénieurs du site de Vergèze à partir à l'étranger pour des missions de repérage ou de conseil technique.

Il reste que les relations entre l'ancien et le nouveau centre furent essentiellement marquées par des importations sur le site vergèzois de pratiques nouvelles - dans le domaine technique mais surtout managérial -, qui remirent en cause l'équilibre des forces instauré du temps de la prospérité antérieure à l'affaire du benzène. Ainsi, dans les années 1990, le site vergèzois a fait l'objet d'une reconfiguration multiforme, effectuée à plusieurs échelles, des unités de production aux lignes d'assemblage. Loin d'être géographiquement neutre, l'espace de travail évolue sous l'effet de contraintes économiques et sociales. Ces modifications prenaient en compte un double souci - et suggèrent par là même la double dimension du fonctionnement industriel, à la fois technique et sociale ${ }^{13}$. D'une part, il s'agissait de répondre aux contraintes techniques de production et à la situation du marché globalisé des eaux embouteillées ${ }^{14}$. Ainsi la spécialisation des trois ateliers, qui effectuaient auparavant les mêmes tâches, est-elle censée répondre à l'objectif de " flux tendu » que se fixe la direction, afin de gagner en réactivité sur un marché agro-alimentaire très réactif aux évolutions de la demande. D'autre part, l'espace de l'usine constitue le cadre géographique au sein duquel évoluent les ouvriers, seuls agents à être en contact direct avec le produit. En modifiant les locaux, il s'agissait pour la direction d'adapter ces "opérateurs ", leurs gestes et leurs déplacements, aux conditions nouvelles de la

12. M. Espagne, Les transferts culturels franco-allemands, Paris, PUF, 1999.

13. G. Rот, Sociologie de l'atelier. Renault, le travail ouvrier, le sociologue, Toulouse, Octarès, 2006 ; "Fluidité industrielle, fragilité organisationnelle ", Revue Française de Sociologie, octobre-décembre 2002, p. 711-737 : "C'est sur une base technique hétérogène, organisée selon une logique de flux tendus, que prennent place, non sans tensions, les moyens de mobilisation et de contrôle des opérateurs à qui incombe la responsabilité d'assurer la continuité productive. Le fonctionnement industriel est le produit de l'articulation entre ces différents niveaux techniques et sociaux organisant la fluidité de la production."

14. N. MarTy, «L'eau embouteillée : histoire de la construction d'un marché », Entreprises et Histoire, avril 2008, p. 86-99. 
production des bouteilles Perrier. Un cas, extrême, mérite l'attention : les murs et le plafond d'un des ateliers de l'usine, auparavant gris, furent entièrement repeints en blanc. Comment interpréter une telle mesure, dont l'efficacité productive semble sujette à caution ? Les recherches réalisées suggèrent que dans l'esprit des nouveaux dirigeants, la nouvelle blancheur des murs devait inspirer aux salariés le « respect du produit ». Investie d'une mission de communication, la peinture de l'atelier était chargée d'illustrer l'ordre nouveau qui devait régner à l'usine - ordre productif mais aussi ordre hiérarchique.

L'espace de production gagne dès lors à être resitué dans une perspective historique, car il constitue en lui-même un enjeu de luttes sociales ${ }^{15}$. À la Source Perrier, sa réorganisation préfigure en partie la remise en cause de l'autonomie ouvrière à l'usine, une tentative de reprise en main partielle du contrôle patronal sur les corps.

\section{Transformer le travail, contrôler davantage les corps : vers un regain de pouvoir patronal sur le site vergèzois}

Les gestes, les déplacements, les attitudes laissent peu de traces écrites, et ceci rend délicat l'accès de l'historien aux corps - difficulté méthodologique soulignée à maintes reprises par l'historiographie ${ }^{16}$. Toutefois la lecture de certaines archives (rapports de Comités d'Hygiène, Sécurité et Conditions de Travail [CHSCT] notamment) et certains témoignages recueillis auprès d'anciens salariés laissent deviner les pratiques en vigueur dans les divers ateliers et sur le lieu de travail en général. Il apparaît ainsi que les salariés de Perrier ont conquis au fil du temps un certain degré d'autonomie, dans les domaines hautement disputés que sont l'espace et le temps au travail. Par exemple, les déplacements sur le site étaient nombreux et se faisaient assez librement ; de même, certains ouvriers jouent sur les cadences, qu'ils augmentaient en dépit des contraintes de sécurité - même recommandées par les syndicalistes membres du CHSCT - pour libérer ensuite un temps libre qu'ils consacraient à diverses pratiques de sociabilité telle que la conversation, ou les jeux de cartes. Cette marge importante d'autonomie s'explique par deux facteurs principaux. Tout d'abord, les ouvriers avaient longtemps bénéficié d'une position de relative force sur le marché du travail local. L'activité agricole y est restée assez importante, même après 1945 : en Vaunage, la viticulture ne connaissait pas (encore) de grave crise, et la taille moyenne des exploitations assurait aux agriculteurs un niveau de vie assez confortable. Dès lors le travail à l'usine ne fournissait qu'un salaire d'appoint : même dans les années 1970, nombreux étaient les ouvriers qui désertaient l'usine en septembre pour aller aider aux vendanges. L'audience de la CGT-Perrier, qui a adopté une ligne plus offensive à partir des années 1960, est un autre facteur expliquant le degré important d'autonomie ouvrière à l'usine. Mai 1968 a marqué à ce titre une véritable rupture ${ }^{17}$ : plus encore qu'une série de conquêtes sociales, plusieurs anciens salariés et militants décrivent cet événement comme une "libération de la parole ", un regain de confiance de la population ouvrière vis-à-vis de la direction. Dans ce contexte, la production de Perrier à l'usine jusqu'au rachat par Nestlé a reposé

15. G. NorRIel, «Espace de production et luttes sociales : l'exemple des usines sidérurgiques lorraines 1880-1930 ", Le Mouvement Social, octobre-décembre 1983, p. 25-56.

16. A. Corbin, J.-J. Courtine, G. Vigarello (dir.), Histoire du corps, t. II : De la Révolution à la Grande Guerre, Paris, Le Seuil, 2005.

17. Cf. X. VIGNA, L'Insubordination ouvrière dans les années 1968. Essai d'histoire politique des usines, Rennes, Presses Universitaires de Rennes, 2007. 
sur un compromis entre une direction soucieuse de disposer d'une main-d'œuvre suffisante et une population ouvrière bénéficiant dès lors d'une certaine liberté de mouvement et de gestion de son temps.

C'est ce degré d'autonomie que la nouvelle direction juge problématique. La liberté de déplacement des ouvriers sur le site heurta les conceptions des nouveaux managers, qui se fixèrent pour objectif de reprendre le contrôle sur ces allées et venues. Un tracé fut établi, balisé à la peinture, et justifié par des considérations de sécurité - mesure qui apparaît bien comme une tentative de reprise de contrôle patronal sur les corps des ouvriers, de regain de pouvoir au sens foucaldien du terme. On aurait certes tort de surestimer la marge de manœuvre disciplinaire de la direction ${ }^{18}$, ou de réduire les corps ouvriers à de simples réceptacles subissant passivement le pouvoir venu "d'en haut ». Les corps travaillant apparaissent, en revanche, comme des enjeux cruciaux ; et la décennie 1990 comme une période de rupture, de remise en cause du compromis précédent. Toutefois cette remise en cause patronale de l'autonomie ouvrière ne s'est pas uniquement traduite par des mesures ponctuelles de balisage de l'espace ou de peinture des murs des ateliers.

C'est à une vaste refonte du " système social Perrier ${ }^{19}$ que s'est employée la direction afin d'obtenir ce regain de pouvoir à l'usine. Plusieurs dispositifs furent instaurés, qui concernèrent tout d'abord l'organisation du travail, désormais placée sous le signe d'une notion revisitée, celle d'autonomie. Augmentation de la polyvalence des ouvriers sur la chaîne de travail, diminution des niveaux hiérarchiques, regroupement des ouvriers en équipes, objectif de juste-à-temps sont autant

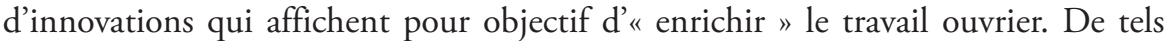
discours illustrent la prise en compte par les acteurs du capitalisme contemporain de ce qu'Eve Chiapello et Luc Boltanski ont dénommé sa " critique artiste " ${ }^{20}$. De plus, les modes de rémunération furent guidés par des principes nouveaux. Plusieurs mesures introduisirent par exemple une dose de rémunération au mérite. De même, l'essor de la négociation individuelle entre le salarié et son supérieur hiérarchique consacra peu à peu la montée en puissance du contrat de travail, au détriment de la convention collective : les pratiques reflétaient de ce point de vue une évolution consacrée dans l'ordre juridique français en matière de droit du travail ${ }^{21}$. Autre modification majeure, les règles et le principe du recrutement de saisonniers furent notablement modifiés. La nouvelle équipe dirigeante remit tout d'abord en question le droit de regard tacite qu'avait acquis la CGT-Perrier sur le système d'embauche. Le statut juridique de ces travailleurs évolua - le saisonnier pouvant auparavant raisonnablement espérer un CDI fit désormais place à un travailleur recruté en CDD sans garantie d'embauche. La question du type de contrat de travail adopté

18. G. NoIRIEL, "Du "patronage" au "paternalisme” ", art. cit. : l'auteur y met en garde contre certaines lectures abusives de Foucault et leur application à l'histoire sociale du travail.

19. N. Marty, Perrier, c'est nous! Histoire de la Source Perrier et de son personnel, Paris, Éditions de l'Atelier, 2003.

20. E. Chiapello et L. Boltanski, Le nouvel esprit..., op. cit.

21. J. Borenfreund, A. Lyon-Caen, M-A. Souriac, I. Vacarie, La négociation collective à l'heure des révisions, Paris, Dalloz-Sirey, 2005. Pour une approche historique sur longue période, cf. A. Cottereau, «Droit et bon droit. Un droit des ouvriers instauré puis évincé par le droit du travail (France, XIX siècle) ", Annales HSS, novembre-décembre 2002, p. 1521-1557 et S. DEAKIn, "Legal origin, juridical form and industrialization in historical perspective: the case of the employment contract and the joint-stock company", Socio-Economic Review, January 2009, p. 35-65. 
est donc traversée par des enjeux majeurs, dépassant de loin le seul cadre juridique. L'évolution de la nature des contrats de travail conclus à la Source Perrier traduit en effet une inflexion considérable des politiques de gestion des « ressources humaines ». Le type de contrat choisi diffère selon la nature des détenteurs du pouvoir en la matière : la substitution de la fonction $\mathrm{RH}$ au précédent droit de regard de la CGT-Perrier suggère une indéniable reprise en main patronale à l'usine.

\section{La judiciarisation des conflits sociaux}

Signe de la tension qui s'installe à l'usine en temps de crise, les différends juridiques prennent une importance grandissante à la Source Perrier au cours des années 1990. Les relations sociales s'en trouvent affectées à deux titres. D'une part, un nombre croissant de conflits se résout au tribunal, phénomène de " judiciarisation " qui suscite en partie, et à l'occasion, un déplacement du centre de gravité des luttes depuis l'usine vers le tribunal. Par conséquent, l'éventualité d'un litige amène d'autre part les acteurs à attacher une plus grande attention aux problèmes de droit, phénomène de " juridicisation " qui a aussi son importance puisque l'univers symbolique dans lequel les acteurs évoluent est de plus en plus imprégné de références juridiques.

\section{Quand les luttes glissent de l'usine vers le tribunal : la judiciarisation partielle des conflits sociaux}

Un nombre important de conflits sociaux fut en partie résolu hors de l'usine. Que le tribunal apparaisse comme un lieu de dénouement crédible de certains différends entre direction et salariés, comme le relais potentiel d'une négociation défectueuse, ne constitue pas en lui-même un changement notable. L'histoire du mouvement social français depuis le XIX ${ }^{\mathrm{e}}$ siècle suggère que les revendications portées par les syndicats comportaient une dimension juridique ${ }^{22}$, dont les dispositions devaient s'ajouter à des perspectives de changement social à plus long terme, conformément au programme proposé aux travailleurs par la Charte d'Amiens. Dans les représentations ouvrières, les avancées en droit occupaient une place secondaire ${ }^{23}$ mais non négligeable, de sorte que la loi et, dans une moindre mesure, le tribunal faisaient figure de "possibles ". L'histoire sociale de la Source Perrier est également faite d'incursions dans la sphère juridique : Nicolas Marty a montré à quel point le syndicaliste René Delon et le juriste Jean Meloux avaient été actifs dans les affaires survenues à la Source sous la précédente direction ${ }^{24}$.

La décennie 1990 à la Source Perrier marque en revanche une inflexion quantitative : à partir du rachat de la Source Perrier par Nestlé, le nombre des contentieux s'accroît notablement. La négociation de mesures telles que les plans de réduction des effectifs échoua à plusieurs reprises, et donna lieu à un litige traduit devant le

22. A. Supiot, Critique du droit du travail, Paris, PUF, 2007 (1994).

23. F. Soubiran-Paillet, "Recours à des catégories juridiques et ressources de protagonistes dans un conflit du travail ", Droit et Société, n 1, 1989 ; Le recours à la justice dans les conflits du travail: histoire d'un détour (l'exemple de l'automobile), Paris, CESDIP, 1987. L'auteur y démontre que les stratégies judiciaires ne sont pas la priorité des militants et conclut à l'absence de "solution dominante dans le rapport entre le conflit et le litige».

24. N. Marty, «Les conditions historiques d'un revirement de jurisprudence majeur dans l'histoire du droit du travail français : les arrêts Perrier de la cour de Cassation (1974) ", in D. Barjot (dir.), Le travail et les hommes : le travail à l'époque contemporaine, actes du $127^{\mathrm{e}}$ Congrès national du CTHS, Paris, Éditions du CTHS, 2004 ; Perrier, c'est nous!..., op. cit. 
tribunal de grande instance de Nîmes. L'enchaînement des faits menant au procès suit un schéma récurrent : la direction présente son projet devant les membres du Comité d'établissement, un débat hautement conflictuel s'ensuit, le CE vote un avis défavorable, les représentants des salariés accusent la direction de ne pas respecter certaines dispositions du Code du Travail et décident d'attaquer la mesure en justice, ce qui donne lieu à un litige et à un règlement judiciaire du conflit.

Mais l'évolution est surtout qualitative : alors qu'avant le rachat, ces conflits restaient localisés, après 1990 ils connaissent une publicité nouvelle et assez inattendue. Les litiges opposant les salariés à la direction gagnent en importance et, souvent, dépassent la sphère locale pour "faire jurisprudence " et se voir conférer, par là même, un certain statut d' " exemplarité " aux plans national et communautaire. L'affaire dite " des palettes " illustre ce "saut d'échelle " qu'effectue la Source Perrier à la faveur du traitement judiciaire de certains conflits. Le tournant juridique auquel la résolution de ce litige a donné lieu est régulièrement souligné par les commentateurs du droit du travail français ${ }^{25}$. Devant le souhait de la direction d'externaliser la mise en palettes des bouteilles, les comités d'établissement de la Source Perrier et de Vittel contestèrent son droit à transférer les salariés concernés vers une entreprise sous-traitante, et poussèrent l'institution judiciaire à préciser les conditions d'application de l'article L122-12 concernant le transfert des contrats de travail. Dans un arrêt du 18 juillet 1998, la Cour de Cassation rappela que ce transfert ne peut se faire qu'en cas de transfert d'une " entité économique autonome ", c'est-à-dire " un ensemble organisé de personnes et d'éléments corporels ou incorporels permettant l'exercice d'une activité économique poursuivant un objectif propre ». Selon les magistrats, l'activité Centre bois de la Source Perrier ne relevait pas de cette définition en raison de l'absence de comptabilité propre à cette activité. Cet " arrêt Perrier ", ainsi qu’il y est communément fait référence, illustre à double titre le regain d'intérêt des salariés et de leurs représentants vis-à-vis du règlement judiciaire de certains conflits. En amont, la tenue du procès constituait un sujet de mobilisation aisément identifiable, permettant de coaliser les énergies autour d'une cause commune, dont l'issue - le verdict - était clairement délimitée dans le temps. Le risque de dilution des énergies, encouru notamment en cas de grève, était moins important, et les témoignages oraux recueillis auprès de militants suggèrent la place non négligeable que ces procès occupent, de par leur lisibilité, dans la mémoire collective militante. En aval, l'issue du procès, cette fois favorable, constitua une victoire à laquelle nulle réunion du CE n'aurait espéré aboutir.

Le champ judiciaire apparaît ainsi comme le théâtre d'une conflictualité que le " dialogue social ", dans l'impasse, ne parvenait plus à contenir. Doit-on attribuer cet échec à des dysfonctionnements conjoncturels, et aisément amendables, du cadre de la négociation ? Plusieurs éléments invitent à une conclusion différente : la régularité du schéma d'enchaînement des faits menant au procès suggère que les acteurs étaient plus enclins à une résolution judiciaire, souvent longue et coûteuse, qu'à un quelconque compromis ; et la consultation des archives, notamment des comptes rendus de réunions du $\mathrm{CE}$, révèle un degré de conflictualité peu conciliable avec toute inclination au "dialogue social ». La judiciarisation des conflits sociaux à la Source Perrier semble dès lors le signe d'une opposition radicale et ouverte entre la

25. A. Supiot, A. Jeammaud et J. Pélissier, Droit du travail, Paris, Dalloz, 2006 ; J.-E. RaY, Droit du travail, droit vivant, Paris, Liaisons, 2007. 
direction et les salariés, qu'aucune des parties en présence n'avait l'intention de nier. Le glissement vers la sphère judiciaire constitue une remise en question du bienfondé de l'idéal de " partenariat social » promu aux plans national et transnational.

\section{Présence du droit au quotidien : la "juridicisation" des relations professionnelles}

À l'usine vergèzoise, cette fréquence des litiges et l'importance nouvelle qu'ils acquirent eurent des conséquences notables sur le fonctionnement des institutions représentatives du personnel. L'éventualité d'un procès, de plus en plus crédible, affecta le langage et les représentations des militants de la Source Perrier : une technicité juridique croissante caractérisa progressivement les échanges entre représentants du personnel et de la direction. Cette évolution s'explique certes en partie par l'élévation générale du niveau d'éducation ; mais elle traduit également l'intérêt relativement nouveau que représentait la maîtrise du droit et du débat expert aux yeux des militants syndicaux et des représentants du personnel. L'issue parfois favorable des affaires menées devant le tribunal les amena à réévaluer partiellement leur rapport au droit et à infléchir certaines de leurs stratégies de lutte. Si les questions juridiques n'avaient pas été négligées par le syndicat dans les années précédant le rachat, elles étaient toutefois demeurées confinées au cercle relativement restreint des militants prêts à y consacrer leur temps et leur énergie. Le témoignage d'André Ollier, militant syndical CGT, plusieurs fois représentant du personnel et ancien secrétaire du $\mathrm{CE}$, suggère cette nette évolution :

Dans les relations sociales, il est évident que si on n'a pas la maîtrise du droit, il est très difficile de pouvoir [...] s'affirmer. Il ne s'agit pas de vouloir jouer les avocats, nous n'en sommes pas, mais il s'agit quand même d'avoir une bonne approche des problèmes juridiques. Et je crois qu'un militant se doit de travailler au moins à ce qui fait son quotidien : les relations contractuelles, le droit des institutions représentatives du personnel, CE, CHSCT. Parce que si on n'a pas ces bases, on ne peut pas répondre: " ah non, ça, j’ai pas à vous le donner ; ça, c'est votre obligation ", etc.

La connaissance du droit fut ainsi perçue moins comme le fruit d'un choix délibéré que comme un outil nécessaire à la poursuite des luttes sociales, une compétence permettant de s'extraire de l'isolement du cas particulier local, un savoir-pouvoir, enfin, sans lequel la remise en cause de la parole patronale semblait désormais un rêve pieux. La maîtrise des enjeux juridiques, alliée à une analyse de la situation économique globale, autorisa même les militants salariés de la Source Perrier à être à l'origine d'une enquête parlementaire en 1998. Celle-ci, rendue possible par le soutien d'élus locaux, notamment le député socialiste Alain Fabre-Pujol qui présida la commission, affichait l'ambition d'étudier au plan national «certaines pratiques des groupes nationaux et multinationaux [...] et leurs conséquences sur l'emploi et l'aménagement du territoire ». Elle procéda notamment à un examen des comptes de dix groupes, parmi lesquels Nestlé France et Perrier Vittel ${ }^{26}$, ainsi qu'à l'audition de leurs directions et des représentants de leur personnel. Au cours de leur intervention, ces derniers firent de leur compétence juridique un usage relativement inédit, puisqu'il s'agissait ici du volet législatif, et non judiciaire, du droit. Ils suggérèrent au législateur des pistes de protection des salariés : contrôle des fonds alloués aux

26. L'enquête se penche aussi sur les groupes Rhône-Poulenc, Usinor, Moulinex, IBM France, Hewlett Packard France, Promodès, Crédit Agricole, Bolloré. 
entreprises en cas de restructurations (FNE notamment), protection des marques (projet de labellisation AOC), agrandissement des compétences des institutions représentatives, création de comités d'entreprise mondiaux, recours à l'assistance d'experts au niveau du comité d'entreprise européen. La compétence et l'imagination juridique des représentants des salariés de la Source Perrier leur permirent de conférer à leur action une dimension proprement politique, au sens où l'entend le politiste Paul Pierson ${ }^{27}$.

Au quotidien néanmoins, c'est davantage la menace du procès que celle d'une loi protectrice dont usèrent les militants. La crispation des échanges entre direction et représentants du personnel se traduisit par le souci des différents acteurs de souligner le bien-fondé juridique de leurs démarches, ce qui donna lieu à des interprétations concurrentes de la législation du travail. Un cas illustre particulièrement les enjeux que recouvre cette attention portée aux textes, celui de l'article L 321-4 du Code du Travail. Le dispositif que cet article énonce rend obligatoire l'information et la consultation des représentants du personnel en cas de mise en ouvre d'un plan de sauvegarde de l'emploi. Le délit d'entrave que constitue le non-respect de cette obligation fut certes un motif récurrent de saisine des autorités judiciaires par le comité d'entreprise ; toutefois les représentants du personnel s'en servirent davantage comme d'un prétexte, seul matériau juridique permettant d'interrompre temporairement la marche des plans sociaux. L'affaire étant jugée sur la forme et non sur le fond, il ne pouvait s'agir de discuter au tribunal du bien-fondé du projet, tout au plus d'en ralentir le rythme ; et pourtant l'insistance avec laquelle la direction dénonça régulièrement le recours à l'article L 321, dans la presse notamment, suggère que la menace d'un recours en justice ne fut pas sans effet. Ces querelles autour du délit d'entrave surprennent par leur mélange de formalisme et d'âpreté. Elles suggèrent la vivacité du conflit opposant les représentants de la direction à ceux du personnel, et on aurait sans doute tort d'en limiter l'analyse en restreignant l'usage du droit à un simple processus d'intervention au service de luttes purement conjoncturelles. Il nous semble en effet que l'usage récurrent de l'article L 321-4 traduit moins un formalisme procédurier, auquel les représentants du personnel se rattacheraient avec l'énergie du désespoir, qu'une tentative, modeste mais persévérante, de remise en question du pouvoir patronal à l'usine. À travers les références faites à la catégorie juridique du délit d'entrave, c'est dans un conflit de légitimités que s'engagent les acteurs.

L'importance croissante du droit, au tribunal et au quotidien, souleva toutefois un certain nombre de doutes chez les militants. La nature profondément ambiguë du droit du travail n'avait, à vrai dire, jamais cessé de poser problème aux militants cégétistes, méfiants vis-à-vis d'un mode de règlement des conflits promu à l'origine par des militants issus des milieux catholiques sociaux et soucieux de réconciliation sociale $^{28}$. À l'heure où les solidarités sur le lieu de travail étaient mises à mal par les

27. P. Pierson, Building the activist state : policy development in the United States, 1960-2000, à paraître. L'analyse du champ politique ne saurait retenir comme acteurs les seuls électeurs : les organisations, salariées ou patronales, gagnent à être perçues comme des acteurs politiques dont on aurait tort de négliger l'importance.

28. Y. Guin, "Au coeur du libéralisme : la loi du 22 mars 1841 relative au travail des enfants employés dans les manufactures, usines ou ateliers ", in J.-P. Le Crom (dir.), Deux siècles de droit du travail, Paris, Éditions de l'Atelier, 1998. 
nouvelles mesures d'individualisation des parcours professionnels, et où le rôle des syndicats se trouvait remis en cause dans le champ intellectuel, le recours croissant au droit n'était-il pas suspect de déstabiliser l'action collective sur le lieu de travail, en imposant une résolution des conflits dans un lieu tiers ? Le filtre du langage et des règles de fonctionnement de l'institution judiciaire ne donnait-il pas lieu à une conflictualité orchestrée, et dès lors épurée de sa capacité de subversion et de remise en cause plus globale ? C'est à ce type de questions que la CGT-Perrier fut confrontée tout au long de la décennie. Si au quotidien ses méthodes de lutte faisaient davantage de place à l'outil juridique, le scepticisme restait de mise vis-à-vis d'un instrument suspect de canaliser le conflit et de le réinsérer dans un système politique et économique non cautionné - suspect, autrement dit, de mener à un abandon des principes d'une Charte d'Amiens dont les idéaux animaient encore les pratiques des militants.

\section{Du local au global, la redéfinition des identités sur le site : la culture au centre de luttes sociales}

Les restructurations opérées sur le site de la Source Perrier n'ont pas eu pour seul effet d'amener les acteurs sociaux à infléchir leurs stratégies de lutte ; elles ont également donné lieu à une véritable redéfinition des identités des divers groupes socioprofessionnels en présence, dans un va-et-vient entre les sphères locale et globale ${ }^{29}$. L'ampleur du conflit survenu durant la décennie 1990 amena les acteurs sociaux à revisiter la mémoire collective et à en donner des interprétations concurrentes ; il surprend par la dimension identitaire qu'il a revêtue, au point que c'est au nom de la «culture » qu’y furent menées les luttes sociales. Le " cas Perrier " soulève ainsi de profonds enjeux mémoriels, appelle un "jeu d'échelles " ${ }^{30}$ entre le local et le global, et invite à réexaminer l'usage controversé de la notion de culture sur le lieu de travail.

\section{La nouvelle équipe dirigeante : la construction d'une identité mondialisée}

Revendiquant une identité dépassant largement les limites du site de production vergèzois, le groupe Nestlé s'exposait dès lors à une critique soulignant l'importance de l'ancrage territorial et de l'authenticité du produit. La diversification des secteurs d'activité du groupe, ainsi que l'étendue géographique de son implantation lui interdisaient en effet de se revendiquer d'un quelconque «terroir » ou de " racines " communes, et rendaient plus délicates la mobilisation du groupe dirigeant, la justification de ses pratiques, et l'expansion du groupe. Dès lors l'image des managers de Nestlé fit l'objet d'une réelle attention, et donna notamment lieu à l'ouvrage d'Helmut Maucher précédemment cité : aux dires de ses promoteurs, une véritable " culture d'entreprise " ${ }^{31}$ était censée animer les membres du groupe Nestlé. Ainsi la nouvelle équipe dirigeant, largement renouvelée, revendique une identité résolument globalisée. La localisation des nouveaux centres de décision, désormais géographiquement découplés des unités de production, encourage ce jeu d'échelle patronal : le siège social de Nestlé Waters France est en banlieue parisienne, et le

29. I. FAVIER, Perrier-Nestlé, histoire d'une absorption : histoire sociale d'une entreprise à l'heure des changements culturels: 1990-2000, Ivry-sur-Seine, Éditions de l'Atelier, 2008.

30. J. Revel (dir.), Jeux d'échelles, la micro-analyse à l'expérience, Paris, Éditions de l'EHESS, 1996.

31. Cf. É. Godelier, La culture d'entreprise, Paris, La Découverte, 2006. 
siège social du groupe Nestlé en Suisse, à Vevey ${ }^{32}$. Cette mise en discours de l'identité patronale va puiser dans le répertoire de la littérature managériale développée au début des années 1980 en réaction contre le modèle taylorien ${ }^{33}$, au nom de «valeurs » centrées sur la « fonction personnel » et puisant dans les théories dites de la motivation et du management " participatif».

Cette promotion de la " culture » du groupe ne constitue pas, ou pas seulement, un discours d'autocélébration sans conséquence : elle implique des pratiques précises de gestion de la main-d'œuvre, qui furent mises en œuvre tant à la Source Perrier que sur les sites de Vittel et de San Pellegrino. Un exemple illustre la traduction concrète des discours identitaires patronaux sur le lieu de travail. Ayant pris acte de la mobilité accrue du capital, des exigences actionnariales croissantes en termes de retour sur investissement, mais également des évolutions des goûts des consommateurs contemporains, "la culture d'entreprise " dont se réclament les dirigeants de Nestlé est porteuse d'un idéal nouveau, dit de "juste-à-temps ». C'est au nom de cet idéal que fut introduit à l'usine un système de production en flux tendus, qui eut pour conséquence l'annualisation du temps de travail à la Source Perrier, mesure censée prendre davantage en compte la saisonnalité de la consommation et de la demande de produits Perrier. La "culture d'entreprise Nestlé " gagne donc à être analysée moins comme une simple ornementation voilant des pratiques peu aisément avouables que comme un discours idéologique complexe, donnant lieu à des pratiques concrètes - autrement dit, comme « un ensemble de croyances partagées, inscrites dans des institutions, engagées dans des actions et par là ancrées dans le réel ${ }^{34}$.

\section{La perte de sens d'un groupe socioprofessionnel : les cadres}

Au cours d'une décennie marquée par des plans sociaux et de longues périodes de tension et de conflits, les cadres s'interrogent également sur leur identité au travail ; mais, pour leur part, c'est dans la douleur que naît ce questionnement. D'une part, les cadres doivent répondre aux attentes de la direction, qui les charge de mettre en œuvre la réduction des effectifs afin d'augmenter la productivité sur le site et d'atteindre un taux de retour sur investissement satisfaisant l'actionnariat. La profession doit ainsi s'adapter à de nouvelles méthodes de travail, notamment les " contrats d'objectif » dont le cadre doit répondre annuellement auprès de sa direction. D'autre part, la fonction d'encadrement à l'usine doit faire face à une population salariée soucieuse de la pérennité des emplois et de l'état de ses conditions de travail. Dans ce contexte, cadres et ouvriers en vinrent à entretenir une relation mouvementée - relation complexe qui ne date certes pas du rachat par Nestlé. À certains égards même, la nouvelle équipe d'encadrement semble mue par une volonté d'apaisement : les témoignages oraux traduisent en effet une prise de distance avec l'attitude de classe adoptée précédemment. Par exemple, nombre d'entre eux refusèrent désormais de concevoir la cantine de l'usine comme le lieu d'affrontement symbolique dont elle faisait figure depuis au moins les années 1960 : plusieurs d'entre eux se dirent ainsi

32. Pour faire l'histoire des multinationales, cf. G. Jones, “Globalization”, in G. Jones et J. ZeitLin (eds.), The Oxford handbook of business history, Oxford, Oxford University Press, 2008, p. 141-168.

33. A. Hatchuel, "Histoire des révolutions de la gestion des entreprises ", Problèmes économiques, $\mathrm{n}^{\circ} 2852,2004$.

34. E. Chiappello et L. Boltanski, Le nouvel esprit..., op. cit. 
choqués de la stricte distinction entre les tables qui leur étaient réservées et celles attribuées au reste des salariés. Est-ce à dire que régnait désormais à l'usine un esprit de partenariat social diffusé jusque dans les espaces de vie quotidienne commune? On peut en douter, étant donné la violence des échanges entre ces deux catégories socioprofessionnelles : au plus fort de la crise, c'est-à-dire durant la discussion des plans sociaux en comité d'entreprise, certains cadres se souviennent avoir été hués à leur entrée à la cantine. La perception qu'ont les cadres de l'espace de restauration commun se prête dès lors à une interprétation, tant elle renseigne sur le nouvel esprit de l'équipe d'encadrement. La prise de distance avec les pratiques jugées rigides des anciens cadres, l'indifférence aux distinctions hiérarchiques trop nettement affichées, l'idéal de dialogue social avec des représentants salariés jugés " responsables », sont autant d'éléments qui indiquent que les nouveaux cadres de la Source Perrier ont repris à leur compte la " critique artiste " du capitalisme contemporain et ont tenté d'infléchir en conséquence leur attitude vis-à-vis des salariés - stratégie toutefois mise à mal par la crise que traverse l'usine.

De part et d'autre du spectre social de l'usine, les cadres voient ainsi leur marge d'autonomie réduite : représentants directs, et visibles, du pouvoir à l'usine face aux salariés, premiers interlocuteurs d'une nouvelle équipe dirigeante qui les charge de mettre en œuvre les restructurations, ils se retrouvent pris dans une inconfortable situation d'entre-deux. Par ailleurs, l'origine géographique de plus en plus variée des cadres contribue à une relative perte de cohésion, certains d'entre eux étant issus d'une promotion interne au sein de l'entreprise et donc originaires de la région, d'autres étant mutés à Vergèze depuis d'autres sites de production. La décennie mouvementée qu'a connue la Source Perrier conduit ainsi à souligner les fissures qui ont pu affecter l'identité et la - déjà toute relative - cohésion de cette catégorie socioprofessionnelle. Catégorie résultant d'une construction sociale récente, entamée durant les années 1930 et plus particulièrement pendant le Front Populaire ${ }^{35}$, la figure sociale du cadre apparait en effet « indissociable d'un certain âge industriel, au cours duquel le paradigme taylorien s'était imposé pour distinguer clairement concepteurs et exécutants " ${ }^{36}$. Les années 1990 à l'usine vergèzoise rappellent que cette catégorie socioprofessionnelle a bel et bien une histoire, et qu'elle est à ce titre susceptible de remises en cause.

L'histoire syndicale se fait l'écho des aléas de la fonction d'encadrement à l'usine. À la Source Perrier, la CGC connaît une histoire assez atypique, marquée par deux périodes distinctes : avant d'être reprise en main par des membres plus en accord avec la "ligne " nationale, la CGC-Perrier soutient pendant plusieurs années des revendications proches de celles de la CGT-Perrier. Une telle stratégie s'explique en partie par les convictions et la personnalité de son principal animateur, enclin à faire cause commune avec les représentants ouvriers et employés. Mais elle suggère également que, jusqu'en 1998, certains cadres syndiqués fondent leur identité

35. L. Boltanski, Les cadres, la formation d'un groupe social, Paris, Éditions de Minuit, 1982. I. Коцвоом, "Patronat et cadres : la contribution patronale à la formation du groupe des cadres (1936-1938) ", Le Mouvement Social, octobre-décembre 1982, p. 71-95. Voir aussi les articles de C. Neumann et N. Hugot-Piron dans le présent numéro du Mouvement Social. Pour la suite, voir notamment les jalons posés par A. GRELON, «Profils d'ingénieurs français (1950-1980) », Le Mouvement Social, avril-juin 1993, p. 85-100.

36. P. Bouffartigue, Les cadres, fin d'une figure sociale, Paris, La Dispute, 2001. 
socioprofessionnelle sur un attachement fort à l'établissement, au risque de s'inscrire en faux contre leurs nouveaux supérieurs hiérarchiques. Après cette date, suite au départ du précédent secrétaire, la CGC-Perrier adopte une stratégie plus soucieuse d'afficher son soutien à la direction et de se distinguer des salariés.

Il reste que ces stratégies et revirements syndicaux successifs traduisent le désarroi certain que traverse la profession, atteinte elle aussi par la crise et le conflit de légitimité en vigueur à l'usine pendant la décennie.

\section{Quand la mémoire ouvrière est au coeur des luttes sociales}

L'identité ouvrière - et employée, dans une moindre mesure - est peut-être celle qui connaît les changements les plus nombreux. Fragmentation des statuts, départs en retraite anticipée des ouvriers les plus expérimentés, recrutement de jeunes à temps partiel pour de courtes durées, exigences accrues en termes de formation initiale, déstabilisent l'identité ouvrière à l'usine et en éliment la mémoire. Paradoxalement, les salariés constituent à la Source Perrier le groupe qui mène à bien le travail de réappropriation de sa mémoire le plus abouti. Confirmant en pratique les thèses de Maurice Halbwachs sur les usages sociaux de la mémoire ${ }^{37}$, un certain nombre de salariés mobilisent le passé à des fins de construction identitaire et de lutte sociale. Ainsi dans les années 1990 s'opère, sous l'égide de la CGT-Perrier essentiellement, un travail de contre-communication dont les archives locales portent de nombreuses traces : les termes de "culture " et d' "identité " font notamment l'objet de redéfinitions. À la culture d'entreprise promue par l'équipe dirigeante, les salariés et leurs représentants des salariés opposent leur vision de l'histoire de l'établissement. La confrontation entre les témoignages oraux de certains salariés suggère que cette histoire commune n'échappe pas à certaines mythifications. Ainsi, les relations professionnelles sont parfois présentées sous un angle particulièrement positif, en omettant de restituer le contexte de luttes dans lequel s'inscrivent les concessions patronales, notamment en matière de salaire - contexte que restitue rigoureusement Nicolas Marty ${ }^{38}$.

Cet investissement de la sphère des représentations par des acteurs sociaux antagonistes culmine dans le débat suscité par le projet de tourisme culturel nourri par la direction, mis en ouvre à partir de 1996. Accusant les coups des campagnes menées par la CGT afin de sensibiliser la région à la situation sociale périlleuse de l'usine, la direction était soucieuse de contrecarrer l'hégémonie culturelle acquise sur le site par le syndicat. Il s'agissait alors de promouvoir une mémoire " alternative " à la Source Perrier, insistant sur l'histoire du produit, de ses succès commerciaux et de ses extravagances publicitaires. Du fait de contraintes budgétaires, ce projet connut un succès inférieur aux attentes initiales de la direction, mais non négligeable pour autant. Les débats qui ont entouré sa mise en œuvre n'en suggèrent pas moins l'actualité des travaux d'E. P. Thompson, qui souligne combien la notion de culture ne saurait être découplée des luttes sociales qui contribuent à ses redéfinitions permanentes ${ }^{39}$.

Le long conflit social qui a divisé et divise la Source Perrier invite ainsi à revisiter à double titre la notion de culture, en mettant en question à la fois certaines

37. M. Halbwachs, La mémoire collective, nouvelle édition, Paris, Albin Michel, 1997.

38. Perrier, c'est nous!..., op. cit.

39. E. P. Thompson, Customs in common : studies in traditional popular culture, Londres, Merlin, 1991. 
lectures abusives de la notion de superstructure chez Marx, qui conduisent à réduire la culture à un simple symptôme, et le culturalisme, qui tourne trop souvent le dos au réel ${ }^{40}$ et envisage la culture comme un domaine strictement autonome, déconnecté des enjeux qui traversent la sphère économique et sociale. Ainsi ne s'agit-il pas ici de réifier les identités des groupes socioprofessionnels, ni de célébrer un ethos dans lequel les membres de ces groupes seraient pris et qui les définirait tout entier. La référence faite dans le présent article à la notion de culture part avant tout de l'usage qu'en font les acteurs mêmes. Au seuil de cette étude, cette notion apparaît davantage comme un instrument de lutte que comme un outil conceptuel entièrement satisfaisant - car le choc des restructurations pousse les acteurs, ouvriers notamment, à aller bien au-delà de la place à laquelle leur « identité professionnelle » semblerait les cantonner. Réappropriation de l'outil juridique, formulation d'un avis expert sur des questions techniques et financières, revendication d'un élargissement des prérogatives du CE : l'histoire des pratiques sociales ouvrières et militantes à la Source Perrier s'apparente bien moins à un repli identitaire sur soi-même qu’à une émancipation intellectuelle ${ }^{41}$. À l'heure où la pérennité de l'usine est remise en question, c'est à un franchissement des frontières, non à une autocélébration, que se sont attachés les ouvriers et les militants de la Source Perrier.

40. V. E. Bonnell et L. Hunt (eds.), Beyond the cultural turn: new directions in the study of society and culture, Berkeley, University of California Press, 1999.

41. J. Rancière, Le Mâttre ignorant. Cinq leçons sur l'émancipation intellectuelle, Paris, Fayard, 1987. 